\title{
STUDYING EPOXY RESIN REINFORCED WITH SILICA DIOXIDE NANOPARTICLES BY MICROINDENTATION
}

\author{
S. V. Smirnov ${ }^{1}$, E. O. Smirnova ${ }^{1}$, I. A. Veretennikova ${ }^{1}$, , V. M. Fomin ${ }^{2}$, \\ A. A. Filippov ${ }^{2}$, T. A. Brusentseva ${ }^{2}$ \\ ${ }^{1}$ Institute of Engineering Science, Ural Branch of the Russian Academy of Sciences, \\ 34 Komsomolskaya st., Ekaterinburg, Russian Federation \\ ${ }^{2}$ Federal State Budgetary Institution of Science \\ Institute of Theoretical and Applied Mechanics. S.A. Khristianovich \\ of the Siberian Branch of the Russian Academy of Sciences, \\ Novosibirsk, Russian Federation \\ *Corresponding autor. E-mail: irincha@imach.uran.ru; \\ address for correspondence: 34, ul. Komsomolskaya, 620049, Ekaterinburg, Russian Federation; \\ tel.: +7 (343) 37535 96; fax: +7 (343) 3745330 \\ The Primer 204 epoxy resin with different contents of silica dioxide nanoparticles is investi- \\ gated by microindentation. The effect of loading time and particles distribution inside the polymer \\ matrix on Martens hardness is examined. The creep of epoxy resin at various hold times is investi- \\ gated. A quantitative relation of the micromechanical properties to the volume content of silica di- \\ oxide nanoparticles is established. The processed experimental data has demonstrated that the test \\ material exhibits viscous properties manifested in the presence of creep.
}

Key words: epoxy resin, silicon dioxide nanoparticles, creep, microindentation.

DOI: $10.17804 / 2410-9908.2017 .1 .024-035$

\section{References}

1. Jumahata A., Soutisb C., Abdullaha S.A., Kasolanga S. Tensile properties of nanosilica/epoxy nanocomposites. International Symposium on Robotics and Intelligent Sensors 2012 (IRIS 2012) Procedia Engineering, 2012, vol. 41, pp. 1634-1640.

2. Starkova O., Buschhorn S.T., Mannov E., Schulte K., Aniskevich A. Creep and recovery of epoxy/MWCNT nanocomposites. Composites: Part A, 2012, vol. 43, pp. 1212-1218. DOI: 10.1016/j.compositesa.2012.03.015.

3. Jia Yu, Peng Ke, Gong Xing-long, Zhang Zhong. Creep and recovery of polypropylene/carbon nanotube composites. International Journal of Plasticity, 2011, vol. 27, pp. 1239-1251. DOI: 10.1016/j.ijplas.2011.02.004

4. Sherif H. Aboubakr, Usama F. Kandil, Mahmoud Reda Taha. Creep of epoxy-clay nanocomposite adhesive at the FRPinterface: A multi-scale investigation. International Journal of Adhesion and Adhesives, 2014, vol. 54, pp. 1-12. DOI: 10.1016/j.ijadhadh.2014.04.003.

5. Díez-Pascual Ana M., Gómez-Fatou Marián A., Ania Fernando, Flores Araceli. Nanoindentation in polymer nanocomposites. Progress in Materials Science, 2015, vol. 67, pp. 1-94. DOI: 10.1016/j.pmatsci.2014.06.002.

6. Oliveira G.L., Costa C.A., Teixeira S.C.S., Costa M.F. The use of nano- and microinstrumented indentation tests to evaluate viscoelastic behavior of poly (vinylidene fluoride) (PVDF). Polymer Testing, 2014, vol. 34, pp. 10-16. DOI: 10.1016/j.polymertesting.2013.12.006.

7. Fascea L.A., Seltzer R., Frontini P.M. Depth sensing indentation of organic-inorganic hybrid coatings deposited onto a polymeric substrate. Surface \& Coatings Technology, 2012, vol. 210, pp. 62-70. DOI: 10.1016/j.surfcoat.2012.08.064.

8. Ngan A.H.W., Wang H.T., Tang B., Sze K.Y. Correcting power-law viscoelastic effects in elastic modulus measurement using depth-sensing indentation. International Journal of Solids and Structures, 2005, vol. 42, nos. 5-6, pp. 1831-1846. DOI: 10.1016/j.ijsolstr.2004.07.018.

Smirnov S.V. et al. / Studying epoxy resin reinforced with silica dioxide nanoparticles

by microindentation 
9. Brusentseva T.A., Filippov A.D., Fomin V.D., Smirnov S.V., Veretennikova I.A. Modification of Epoxy Resin with Silica Nanoparticles and Process Engineering of Composites Based on Them. Mechanics of Composite Materials, 2015, vol. 51, no. 4, pp. 531-538. DOI: $10.1007 / \mathrm{s} 11029-015-9523-6$.

10. GOST R 8.748-2011 (ISO 14577-1:2002). Gosudarstvennaya sistema obespecheniya yedinstva izmereniy. Metally $i$ splavy. Izmerenie tverdosti $i$ drugikh kharakteristik materialov pri instrumentalnom indentirovanii. Ch. 1. Metod ispytaniy. Vved. 2013-05-01 [State System for Ensuring the Uniformity of Measurements. Metals and Alloys. Measurement of Hardness and Other Material Characteristics in Instrumented Indentation. Part 1. Test Method. Introd. 2013-05-01]. M., Standartinform Publ., 2013, 24 p. (In Russian).

11. Rabotnov Yu.N. Polzuchest elementov konstruktsiy [Creep of Structural Elements]. M., Nauka Publ., 1966, 752 p. (In Russian).

12. Malinin N.N. Prikladnaya teoriya plastichnosti i polzuchesti [Applied Theory of Plasticity and Creep]. M., Mashinostroenie Publ., 1975, 400 p. (In Russian).

13. Fischer-Cripps A.C. A simple phenomenological approach to nanoindentation creep. Materials Science and Engineering A, 2004, vol. 385, iss. 1-2, pp. 74-82. DOI: 10.1016/j.msea.2004.04.070.

14. Natsik V.D., Fomenko L.S., Lubenets S.V. Investigation of the creep and glass transition of elastomers by the microindentation method: Epoxy resin and related nanocomposites. Physics of the Solid State, 2013, vol. 55, no. 5, pp. 1020-1033. DOI: 10.1134/S1063783413050260.

Birger I.A., Mavlyutov R.R. Soprotivlenie materialov. Uchebnoe posobie [Strength of Materials. Tutorial]. M., Nauka. Gl. red. fiz.-mat. lit. Publ., 1986, 560 p. (In Russian). 
Подана в журнал: 17.02 .2017

УДК 620.178.152.341.4:620.178.156

DOI: $10.17804 / 2410-9908.2017 .1 .024-035$

\title{
ИССЛЕДОВАНИЕ МЕТОДОМ МИКРОИНДЕНТИРОВАНИЯ ОБРАЗЦОВ ЭПОКСИДНОЙ СМОЛЫ, УПРОЧНЕННОЙ НАНОЧАСТИЦАМИ ДИОКСИДА КРЕМНИЯ
}

\author{
С. В. Смирнов ${ }^{1}$, Е. О. Смирнова ${ }^{1}$, И. А. Веретенникова ${ }^{1 *}$, В. М. Фомин ${ }^{2}$, \\ А. А. Филиппов ${ }^{2}$, Т. А. Брусенцева ${ }^{2}$ \\ ${ }^{1}$ Федеральное государственное бюджетное учреждение науки \\ Институт машиноведения Уральского отделения Российской академии наук, \\ Екатеринбург, Российская Федераиия \\ ${ }^{2}$ Федеральное государственное бюджетное учреждение науки \\ Институт теоретической и прикладной механики им. С.А. Христиановича \\ Сибирского отделения Российской академии наук, \\ Новосибирск, Российская Федерация \\ *Ответственный автор. Электронная почта: irincha@imach.uran.ru; \\ адрес для переписки: 620049, Екатеринбург, ул. Комсомольская, 34, Российская Федерация; \\ тел.: +7 (343) 375-35-96; факс: +7 (343) 374-53-30
}

Методом микроиндентирования исследована эпоксидная смола Праймер 204 с содержанием наполнителя $(1,2,2$ и 3,5 об. \%) из наночастиц диоксида кремния Таркосил Т-20. Изучено влияние продолжительности нагрузки и времени выдержки под нагрузкой на твердость по Мартенсу и ползучесть при микроиндентировании в зависимости от содержания наполнителя. Показано, что вязкоупругая трехзвенная модель Фойгта достаточно точно описывает деформацию ползучести исследованных материалов на стадии выдержки под нагрузкой. Получены эмпирические коэффициенты модели для образцов эпоксидной смолы с разным содержанием наполнителя.

\section{Ключевые слова: эпоксидная смола, наночастиџы диоксида кремния, ползучесть, микроиндентирование.}

\section{1. Введение}

Эпоксидная смола является одной из разновидностей синтетических смол. В чистом виде она не применяется, а приобретает все свои качества при смешивании с отвердителем и завершении реакции полимеризации. Эпоксидные смолы обладают устойчивостью к воздействию кислот, галогенов, щелочей, растворяются в ацетоне и сложных эфирах без образования пленки. Кроме того, они характеризуются высокой прочностью клеевого соединения, минимальной усадкой, незначительной влагопроницаемостью в отвержденном виде, высокой устойчивостью к абразивному износу. Все эти особенности способствовали широкому распространению эпоксидных материалов в качестве основы для адгезивов при изготовлении композитных материалов, покрытий, герметиков, материалов для внутренней и наружной отделки зданий, пропитки для повышения прочности и гидроизоляции пористых материалов и др. Перспективным направлением повышения эксплуатационных характеристик эпоксидных смол является использование добавок-модификаторов различной природы. В результате получается гетерогенный материал, представляющий собой сочетание разнородных компонентов, один из которых образует матрицу (связующее), а другой (наполнитель) обладает высокой прочностью и определенными функциональными свойствами.

При определении возможности применения эпоксидной смолы при изготовлении конкретных изделий необходимо знать ее механические свойства. Теоретические исследования на микро- и макромасштабных уровнях прочностных и упругих свойств полимерных матеSmirnov S.V. et al. / Studying epoxy resin reinforced with silica dioxide nanoparticles

by microindentation 
риалов при наличии добавок проводятся с использованием методов механики сплошных сред, позволяющих описывать как поведение микрообъемов, так и элементов конструкций. Свойства модифицированных полимеров на макромасштабном уровне обычно определяются разработчиками этих материалов в стандартных образцах, подвергаемых испытаниям на растяжение, сжатие, кручение и изгиб [1-4]. Подобный подход технически сложно применить для определения механического поведения материала на микромасштабном уровне, поэтому перспективным является использование метода индентирования [5-6]. Экспериментальные данные обрабатывают с помощью различных методик и получают информацию, пригодную для проведения прочностных расчетов [7, 8 и др.]. В настоящей работе показана возможность применения метода индентирования для изучения локальных механических свойств модифицированной эпоксидной смолы с различным содержанием упрочняющих наночастиц диоксида кремния.

\section{2. Материал и оборудование}

Исследовали эпоксидную смолу Праймер 204 с объемным содержанием 1,2, 2 и 3,5 \% добавки диоксида кремния - Таркосил Т-20. Образцы были изготовлены в Институте теоретической и прикладной механики им. С.А. Христиановича СО РАН и Новосибирском институте органической химии им. Н.Н. Ворожцова СО РАН. В работе [9] были получены исходные данные о влиянии технологии изготовления образцов на их механические свойства. В образцах связующей является эпоксидная смола Праймер-204, характеристики которой приведены в табл. 1.

Таблица 1 - Характеристики эпоксидной смолы Праймер-204

\begin{tabular}{|l|l|}
\hline \multicolumn{1}{|c|}{ Название связующего } & \multicolumn{1}{c|}{ Праймер-204 } \\
\hline Вязкость при $20^{\circ} \mathrm{C}$, МПа·с & 610 \\
\hline Отвердитель & Полиаминный отвердитель \\
\hline Плотность кг $\mathrm{m}^{3}$ & 1100 \\
\hline Соотношение компонентов смеси & $1: 2$ \\
\hline Время высыхания при $\mathrm{t}=20^{\circ}, \mathrm{ч}$ & 15 \\
\hline Цвет & Белый \\
\hline
\end{tabular}

В качестве наполнителя для гетерогенного материала был использован нанопорошок диоксида кремния $\mathrm{SiO}_{2}$ марки Таркосил Т-20 (табл. 2). На рис. 1, полученном на электронном сканирующем микроскопе, видно, что перед изготовлением смеси наполнитель имеет вид агломератов преимущественно в форме неправильных глобулей.

Таблица 2 - Характеристики нанопорошка диоксида кремния Таркосил Т-20

\begin{tabular}{|c|c|c|c|c|}
\hline $\begin{array}{c}\text { Тип } \\
\text { наполнителя }\end{array}$ & $\begin{array}{c}\text { Удельная площадь } \\
\text { поверхности, } \\
10^{3} \mathrm{~m}^{2} / \kappa \Gamma\end{array}$ & $\begin{array}{c}\text { Насыпная } \\
\text { плотность, } \\
\text { кг } / \mathrm{M}^{3}\end{array}$ & $\begin{array}{c}\text { Размер } \\
\text { агломератов, } \\
\text { мкм }\end{array}$ & Цвет \\
\hline Таркосил Т-20 & 135 & $60-70$ & $5-7$ & Белый \\
\hline
\end{tabular}

Smirnov S.V. et al. / Studying epoxy resin reinforced with silica dioxide nanoparticles by microindentation 
hittp://dreqm-journal.or"]

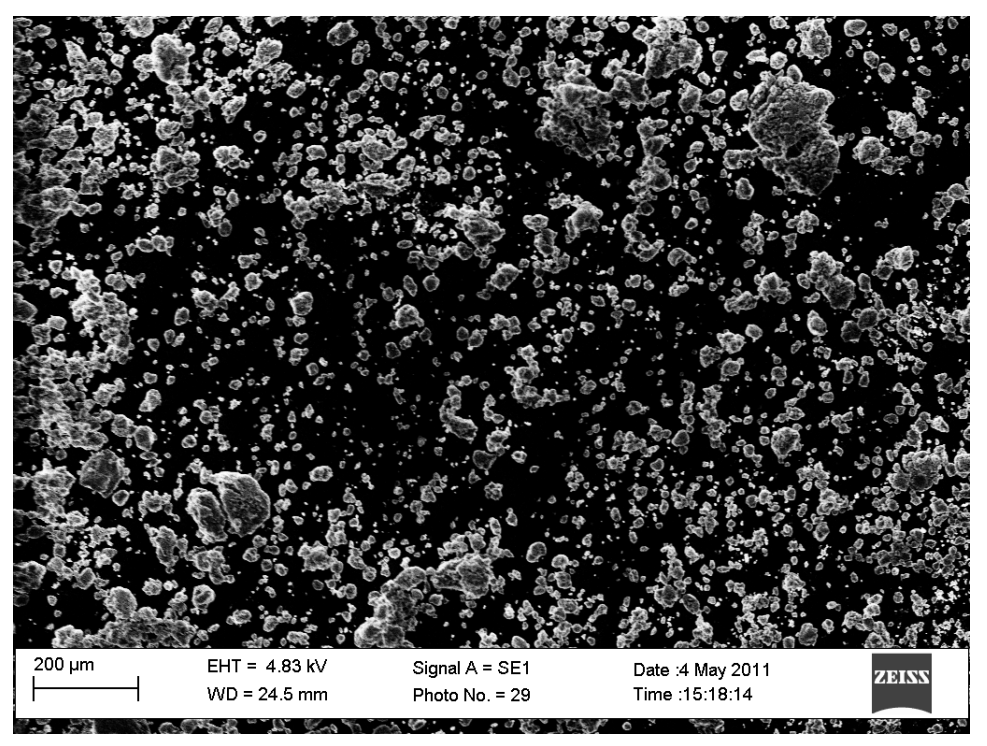

Рис. 1. Частицы Таркосила Т-20

Определение микротвердости проводили с использованием автоматизированной системы для измерения микротвердости FISCHERSCOPE HM2000 XYm (рис. 2). Согласно паспорту прибора, диапазон измерения твердости составляет 0,001-120000 H/Mм², точность задания нагрузки составляет 4 мг, перемещения измеряются с точностью 0,1 нм. Погрешность определения микротвердости составляет $2 \%$ от измеряемой величины. Скорость подвода индентора 2 мкм/с. Диапазон нагрузок при испытании 1-2000 мН. Первичную обработку результатов испытаний осуществляли с помощью программного обеспечения прибора WIN-HCU. B качестве индентора использовали четырехгранную алмазную пирамиду Виккерса с ужесточенными требованиями к геометрии.

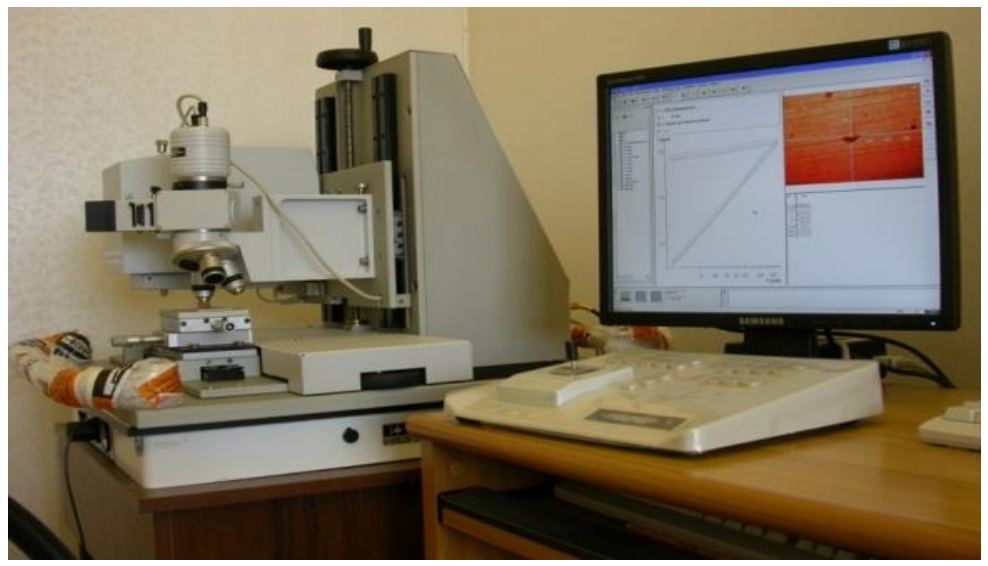

Рис. 2. Система для измерения микротвердости FISCHERSCOPE HM2000 XYm

В качестве характеризующего параметра была выбрана твердость $H M$ по шкале Мартенса. При измерении $H M$ учитывают пластическую и упругую деформации, так что данное значение твердости можно вычислить для широкого круга материалов. Твердость по Мартенсу [10] определяется как отношение текущей величины испытательной нагрузки $F$ к площади поперечного сечения $A_{\mathrm{s}}$ индентора на расстоянии $h$ (глубина внедрения индентора) от вершины и рассчитывается по формуле

$$
H M=\frac{F}{A_{\mathbf{s}}(h)}=\frac{F}{26,43 h^{2}} .
$$

Smirnov S.V. et al. / Studying epoxy resin reinforced with silica dioxide nanoparticles by microindentation 


\section{3. Экспериментальные исследования и их результаты}

Индентирование проводили после отвердевания, на поверхности образца, не подвергнутой механическим воздействиям, что обеспечило отсутствие влияния дополнительной обработки на механические свойства исследуемых материалов. Испытания проводились при температуре $297 \pm 2 \mathrm{~K}$.

Для осуществления индентирования использовали два режима нагружения - треугольный и трапецеидальный (рис. 3). При треугольном режиме нагружения (рис. 3, $a$ ) происходит линейный рост нагрузки и разгрузки с заданным постоянным временем. Трапецеидальный режим нагружения (рис. $3, \sigma)$ характеризуется различным временем выдержки $t_{\text {в }}$ под нагрузкой в сочетании с постоянным временем нагрузки $t_{\mathrm{H}}$ и разгрузки $t_{\mathrm{p}}$.

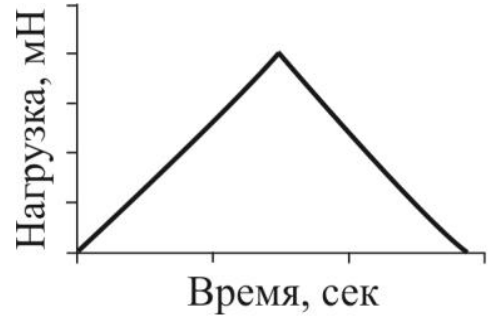

$a$

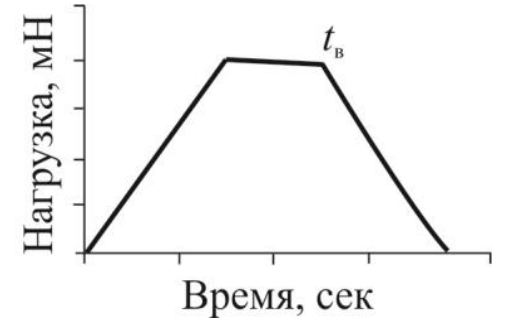

$\sigma$

Рис. 3. Режимы нагружения: $a$ - треугольный, $\sigma$ - трапецеидальный

Существенной технической проблемой при изготовлении полимерных композитов, модифицированных различными частицами, является равномерное распределение наполнителя. В первой серии экспериментов провели оценку степени равномерности распределения твердости на поверхности образца. Для этого проводили многократное индентирование по треугольному режиму нагружения (рис. $3, a$ ) в случайно выбранных местах поверхностей при максимальном усилии $500 \mathrm{MH}$ и времени $t_{\mathrm{H}}=t_{\mathrm{p}}=20 \mathrm{c}$ для всех исследуемых материалов. На рис. 4 в качестве примера полученных результатов приведены диаграммы нагружения для образца с содержанием упрочняющей добавки 1,2 об. \%. Видно, что разные диаграммы нагружения совпадают без значительных смещений. Аналогичная картина наблюдается и для образцов с другим содержанием $\mathrm{SiO}_{2}$. В табл. 3 приведены значения полученных твердостей по Мартенсу для 5 отдельных измерений. На основании проведенных тестов установлено, что разброс в значениях твердости материала для каждого образца остается в пределах 2 \%, что свидетельствует о достаточно равномерном распределении частиц в полимерной матрице.

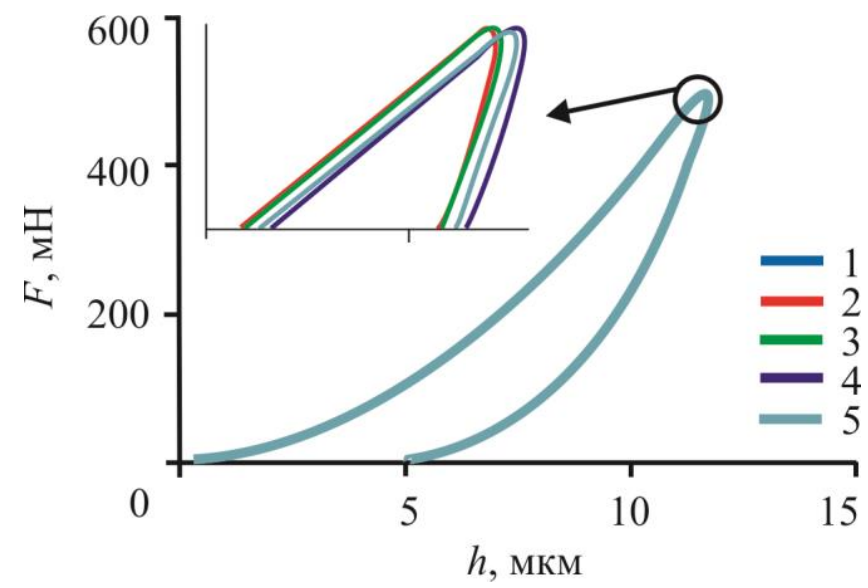

Рис. 4. Диаграммы индентирования при одинаковых условиях для образца с содержанием наночастиц диоксида кремния 1,2 \%, полученные в 5 испытаниях

Smirnov S.V. et al. / Studying epoxy resin reinforced with silica dioxide nanoparticles by microindentation 
Таблица 3 - Значения твердости $Н M$ для образцов с разным содержанием $\mathrm{SiO}_{2}$

\begin{tabular}{|c|c|c|c|c|c|c|}
\hline \multirow{2}{*}{$\begin{array}{c}\text { Содержание } \\
\mathrm{SiO}_{2} \text {, об. \% }\end{array}$} & 1 & 2 & 3 & 4 & 5 & $\begin{array}{c}\text { Среднее } \\
\text { Значение } H M, \\
\text { H/мм }^{2}\end{array}$ \\
\hline 1,2 & 138,69 & 139,75 & 139,59 & 137,97 & 138,62 & 138,92 \\
\hline 2,0 & 136,27 & 135,54 & 136,86 & 136,58 & 136,35 & 136,32 \\
\hline 3,5 & 136,84 & 135,36 & 135,93 & 134,76 & 133,81 & 135,34 \\
\hline
\end{tabular}

Во второй серии экспериментов исследовали влияние продолжительности цикла нагрузки на твердость. Испытания проводили с использованием треугольного режима нагружения (рис. $3, a$ ). Максимальная нагрузка при всех испытаниях составляла $500 \mathrm{mH}$, а время нагрузки составляло $1,5,10,20,30,40,50,60,80$, и 100 с. На рис. 5 ( $a-8)$ приведены

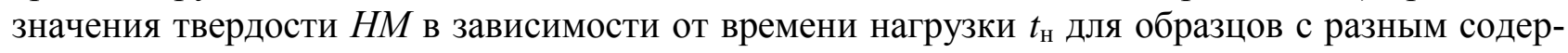
жанием наполнителя. В диапазоне $t_{\mathrm{H}}$ от 1 до 50 с материал проявляет вязкие свойства, выражающиеся в уменьшении твердости при уменьшении скорости приложения нагрузки со 152 до $129 \mathrm{H} / \mathrm{Mм}^{2}$, со 155 до $133 \mathrm{H} /$ мм $^{2}$ и со 158 до $135 \mathrm{H} /$ мм $^{2}$ для образцов с содержанием $1,2,2,0$ и 3,5 об. \% наполнителя, соответственно. Дальнейшее увеличение времени $t_{\text {н }}$ оказывает незначительное влияние на величину твердости.

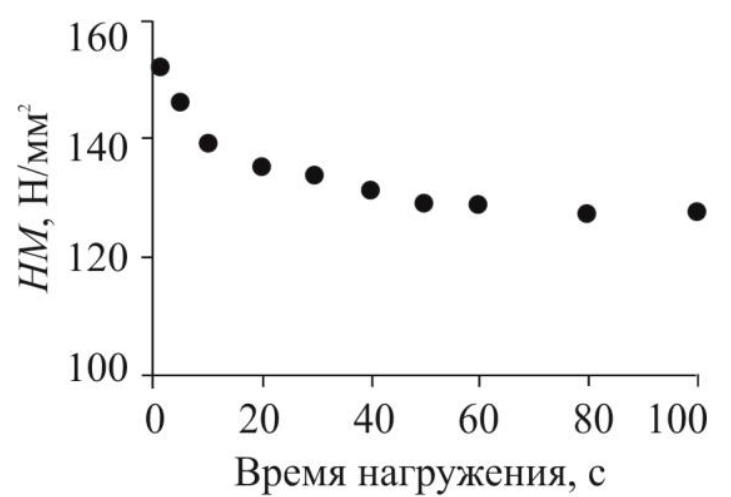

$a$

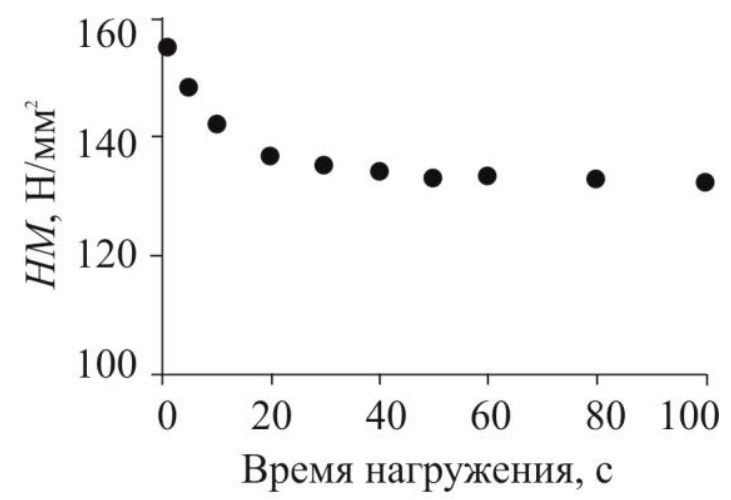

$\sigma$

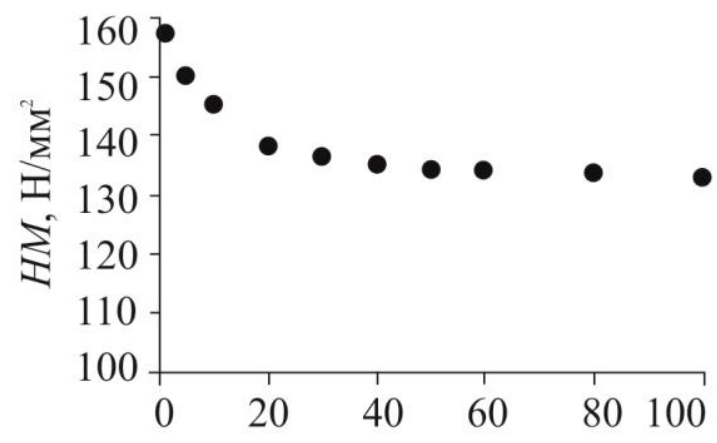

Время нагружения, с

B

Рис. 5. Влияние времени нагружения на твердость $Н M$ по Мартенсу для образцов с содержанием наночастиц диоксида кремния, об. \%: $a-1,2 ; \sigma-2 ; в-3,5$

Важным свойством, отвечающим за искажение геометрических размеров и снижение способности сопротивляться нагрузкам с течением времени, является ползучесть. Для исследования ползучести проводили индентирование по трапецеидальному режиму нагружения Smirnov S.V. et al. / Studying epoxy resin reinforced with silica dioxide nanoparticles 
(рис. 3, б), фиксируя при этом изменение глубины вдавливания индентора в процессе выдержки. В соответствии с ранее проведенными исследованиями в качестве параметров нагружения были выбраны максимальная нагрузка $F=500 \mathrm{MH}$, время нагружения 50 с, время

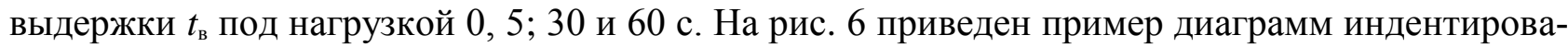
ния для образца с содержанием наполнителя 2 об. \%, а на рис. 7 - средние значения $H M$ для каждого образца при разных $t_{\mathrm{B}}$. Из полученных данных следует, что с увеличением времени выдержки уменьшаются значения твердости и увеличивается глубина внедрения в результате ползучести материала. При выдержке 30 с и выше наблюдается замедление скорости ползучести материала и можно говорить об установившихся значениях $H M$ (релаксированная твердость). Соотношение между релаксированной и начальной твердостью зависит от содержания наполнителя: для образцов с 1,2 об. \% $\mathrm{SiC}$ оно равно 0,996; с 2 об. \% - 0,980; с 3,5 об. \% - 0,976. Этому может быть предложено следующее объяснение. В деформированной в результате вдавливания индентора области материала помимо поля внутренних упругих напряжений, вызванных общей неравномерностью деформации, около твердых частиц наполнителя возникают локальные внутренние напряжения. Чем больше частиц наполнителя содержится в материале, тем больший вклад они вносят в результирующее поле внутренних напряжений. Релаксационные процессы, происходящие во время выдержки под нагрузкой, приводят к уменьшению локальных напряжений, что проявляется в более интенсивном снижении твердости для материала с более высоким содержанием наполнителя относительно его начального значения.

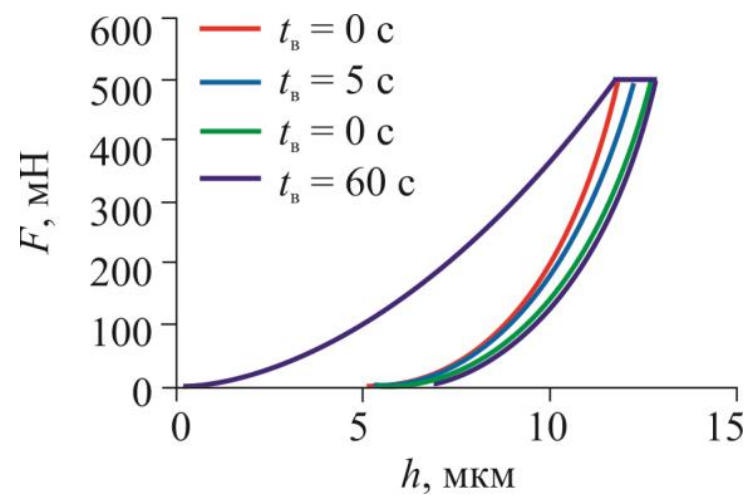

Рис. 6. Диаграммы индентирования для образцов с концентрацией 2 об. \% наполнителя при разном времени выдержки под нагрузкой

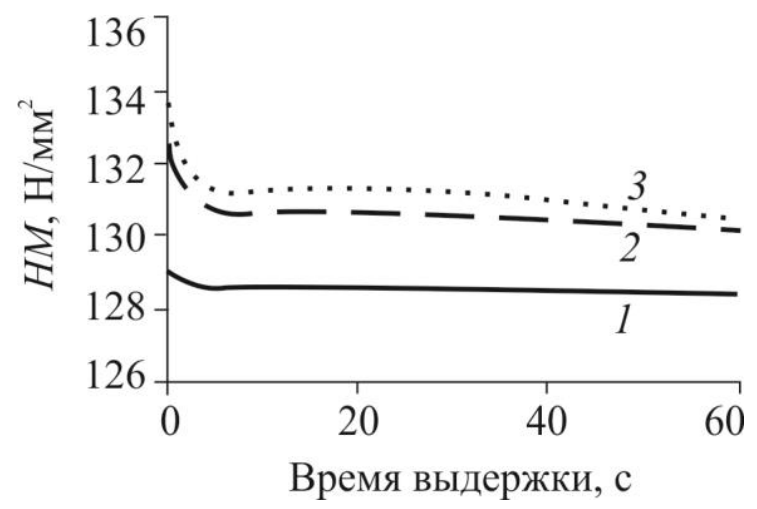

Рис. 7. Изменение твердости $H M$ в зависимости от времени выдержки для образцов с разным содержанием наночастиц $\mathrm{SiO}_{2}$, об. \%: $1-1,2 ; 2-2 ; 3-3,5$

Smirnov S.V. et al. / Studying epoxy resin reinforced with silica dioxide nanoparticles by microindentation 
Для описания ползучести полимеров под действием внешних нагрузок часто используют схематизированные механические модели, представляющие собой различные сочетания элементов в виде идеальных пружин, характеризующих упругие свойства полимера, и демпферов (амортизаторов), характеризующих его вязкие свойства. В научной литературе описан ряд моделей, в которых указанные выше элементы сочетались в различных комбинациях $[11,12]$. В работах $[13,14]$ показана возможность использования механических моделей для описания поведения вязкоупругих материалов в условиях локализованной ползучести при вдавливании жестких инденторов и установлено, что для разных материалов наилучшим образом подходят разные модели. Была оценена возможность описания ползучести исследуемого материала при индентровании с помощью, наиболее часто используемой вязкоупругой трехзвенной модели Фойгта (рис. 8) [15], представляющей последовательное соединение элемента упругости $\left(E_{2}\right)$ и параллельное соединение элементов упругости $\left(E_{1}\right)$ и вязкости $(\eta)$.

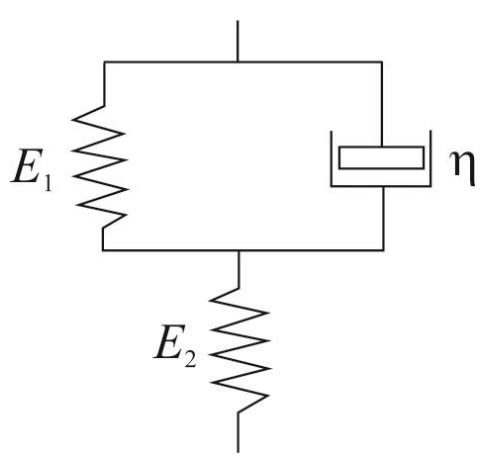

Рис. 8. Механическая вязкоупругая трехзвенная модель Фойгта [15]

Изменение глубины $h$ вдавливания индентора Виккерса под действием силы $F$ в материал, описываемый вязкоупругой трехзвенной моделью Фойгта, определяется следующим уравнением [13]:

$$
h^{2}=F \frac{\pi}{2} \operatorname{ctg} \alpha\left[\frac{1}{E_{1}^{*}}+\frac{1}{E_{2}^{*}}\left(1-\exp \left(-t \frac{E_{2}^{*}}{\eta}\right)\right],\right.
$$

где $E_{1}^{*}=\frac{E_{1}}{1-\mu_{1}^{2}}, E_{2}^{*}=\frac{E_{2}}{1-\mu_{2}^{2}}-$ приведенные нормальные модули упругости для упругих элементов на рис. $8 ; E_{1}, \mu_{1}, E_{2}, \mu_{2}$ - нормальные модули упругости и коэффициенты Пуассона материала упругих элементов; $\eta$ - коэффициент вязкости; $\alpha=70,3^{\circ}-$ эквивалентный полуугол конусности для инденторов Виккерса и Берковича.

Несмотря на ясный физический смысл модели (2) использование ее для расчетов вызывает затруднения из-за присутствия неизвестных значений характеристик упругих свойств и вязкости, которые должны быть определены из экспериментов. Можно сократить количество подлежащих определению параметров, объединив часть из них комплексы следующим образом:

$$
h^{2}(t)=P\left(A_{0}-A_{1} \exp (-t / \tau)\right)
$$

где $A_{0}=\frac{\pi}{2} \operatorname{ctg} \alpha\left(\frac{1}{E_{1}^{*}}+\frac{1}{E_{2}^{*}}\right) ; \quad A_{1}=\frac{\pi}{2} \operatorname{ctg} \alpha \cdot \exp \left(-\frac{t}{\tau}\right) ; \tau=\frac{\eta}{E_{2}^{*}}$.

По мнению авторов работы [14], в таком виде уравнение (3) будет формально представлять интерпретацию процесса ползучести полимера, которая базируется на предположении о протекании в нем конформационного молекулярного процесса с временем релаксации $\tau$.

Smirnov S.V. et al. / Studying epoxy resin reinforced with silica dioxide nanoparticles by microindentation 
Полученные экспериментальные данные по изменению глубины проникновения индентора на стадии ползучести для образцов с разным содержанием наполнителя были подвергнуты нелинейному регрессионному анализу с целью их описания уравнением (3). Полученные значения $A_{0}, A_{1}, \tau$ приведены в табл. 4 . Усредненные экспериментальные зависимости по изменению глубины проникновения индентора на стадии ползучести и полученные аппроксимирующие зависимости изображены на рис. 9.

Таблица 4 - Коэффициенты аппроксимации в модели (3)

\begin{tabular}{|c|c|c|c|}
\hline $\begin{array}{c}\text { Содержание наночастиц } \\
\mathrm{SiO}_{2}, \text { об. \% }\end{array}$ & $A_{0}, \mathrm{мкм}^{2} / \mathrm{MH}$ & $A_{1}, \mathrm{мкм}^{2} / \mathrm{MH}$ & $\tau$ \\
\hline 1,2 & 0,34 & 1,12 & 15,91 \\
\hline 2,0 & 0,33 & 1,16 & 15,62 \\
\hline 3,5 & 0,32 & 1,11 & 15,54 \\
\hline
\end{tabular}

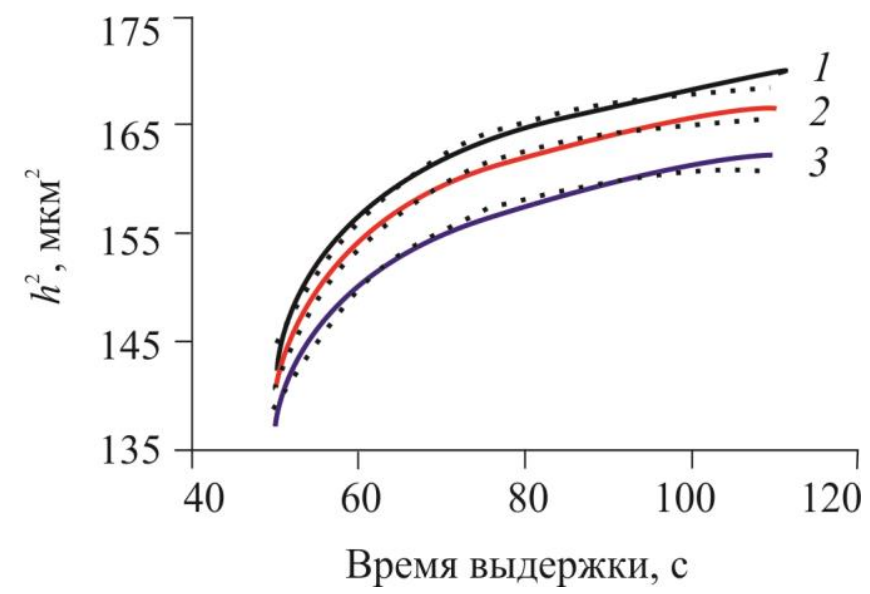

Рис. 9. Усредненные экспериментальные данные (сплошные линии) и аппроксимирующие зависимости (пунктирные линии), полученные для вязкоупругой трехзвенной модели Фойгта.

Содержание наполнителя, об. \%: $1-1,2 ; 2-2 ; 3-3,5$

Полученные результаты показали достаточно хорошую сходимость расчетов по вязкоупругой трехзвенной моделью Фойгта с экспериментальными данными (коэффициент достоверности аппроксимации $\left.R^{2}>0,98\right)$.

\section{Выводы}

Исследования, выполненные методом микроиндентирования на образцах из эпоксидной смолы Праймер 204 с наполнителем из наночастиц диоксида кремния $\mathrm{SiO}_{2}$ марки Таркосил Т-20 при содержании наполнителя 1,2, 2,0 и 3,5 об. \% показали, что:

- разброс значений твердости на поверхности образцов не превышает величины приборной погрешности, что косвенно свидетельствует о равномерном распределении наполнителя в виде агломерированных наночастиц $\mathrm{SiO}_{2}$ в эпоксидной смоле;

- в диапазоне изменения времени нагрузки от 1 до 50 с материал проявляет вязкие свойства, выражающиеся в уменьшении твердости при уменьшении скорости приложения нагрузки со 152 до $129 \mathrm{H} / \mathrm{Mм}^{2}$, со 155 до $133 \mathrm{H} / \mathrm{Mм}^{2}$ и со 158 до $135 \mathrm{H} / \mathrm{Mм}^{2}$ для образцов с содержанием 1,2, 2,0 и 3,5 об. \% наполнителя соответственно; дальнейшее увеличение времени $t_{\text {н }}$ оказывает незначительное влияние на величину твердость;

Smirnov S.V. et al. / Studying epoxy resin reinforced with silica dioxide nanoparticles by microindentation 
- с увеличением времени выдержки уменьшаются значения твердости и увеличивается глубина внедрения индентора в результате ползучести материала; стабилизация величины твердости наблюдается при выдержке под нагрузкой свыше 30 с, при этом эффект снижения твердости тем больше, чем выше содержание наполнителя в образце;

- вязкоупругая трехзвенная модель Фойгта достаточно точно описывает деформацию ползучести исследованных материалов на стадии выдержки под нагрузкой; получены эмпирические коэффициенты модели для образцов эпоксидной смолы с разным содержанием наполнителя.

\section{Благодарность}

Работа выполнена в части разработке технологии изготовления образцов при поддержке гранта РФФИ № 16-31-00135_мол_а, в части исследования ползучести - при поддержке гранта РФФИ № 16-08-01154_а.

\section{Литература}

1. Tensile properties of nanosilica/epoxy nanocomposites / A. Jumahata, C. Soutisb, S. A. Abdullaha, S. Kasolanga // International Symposium on Robotics and Intelligent Sensors 2012 (IRIS 2012) «Procedia Engineering» : Proccedings. - 2012. - Vol. 41. - P. 1634-1640.

2. Creep and recovery of epoxy/MWCNT nanocomposites / O. Starkova, S. T. Buschhorn, E. Mannov, K. Schulte, A. Aniskevich // Composites: Part A. - 2012. - Vol. 43. - P. 1212-1218. DOI: 10.1016/j.compositesa.2012.03.015.

3. Creep and recovery of polypropylene/carbon nanotube composites / Yu Jia, Ke Peng, Xinglong Gong, Zhong Zhang // International Journal of Plasticity. - 2011. - Vol. 27. - P. 1239-1251. DOI: 10.1016/j.ijplas.2011.02.004

4. Sherif H. Aboubakr, Usama F. Kandil, Mahmoud Reda Taha. Creep of epoxy-clay nanocomposite adhesive at the FRPinterface: A multi-scale investigation // International Journal of Adhesion and Adhesives. - 2014. - Vol. 54. - P. 1-12. - DOI: 10.1016/j.ijadhadh.2014.04.003.

5. Nanoindentation in polymer nanocomposites / Ana M. Díez-Pascual, Marián A. GómezFatou, Fernando Ania, Araceli Flores // Progress in Materials Science. - 2015. - Vol. 67. - P. 1-94. DOI: 10.1016/j.pmatsci.2014.06.002.

6. The use of nano- and micro-instrumented indentation tests to evaluate viscoelastic behavior of poly (vinylidene fluoride) (PVDF) / G. L. Oliveira, C. A. Costa, S. C. S. Teixeira, M. F. Costa // Polymer Testing. - 2014. - Vol. 34. - P. 10-16. - DOI: 10.1016/j.polymertesting.2013.12.006.

7. Fascea L. A., Seltzer R., Frontini P. M. Depth sensing indentation of organic-inorganic hybrid coatings deposited onto a polymeric substrate // Surface \& Coatings Technology. - 2012. Vol. 210. - P. 62-70. - DOI: 10.1016/j.surfcoat.2012.08.064.

8. Correcting power-law viscoelastic effects in elastic modulus measurement using depth-sensing indentation / A. H. W. Ngan, H. T. Wang, B. Tang, K. Y. Sze // International Journal of Solids and Structures. - 2005. - Vol. 42, nos. 5-6. - P. 1831-1846. DOI: 10.1016/j.ijsolstr.2004.07.018.

9. Modification of Epoxy Resin with Silica Nanoparticles and Process Engineering of Composites Based on Them / T. A. Brusentseva, A. D. Filippov, V. D. Fomin, S. V. Smirnov, I. A. Veretennikova // Mechanics of Composite Materials. - 2015. - Vol. 51, no. 4. - P. 531-538. DOI: $10.1007 / \mathrm{s} 11029-015-9523-6$.

10. ГОСТ Р 8.748-2011(ИСО 14577-1:2002). Государственная система обеспечения единства измерений. Металлы и сплавы. Измерение твердости и других характеристик материалов при инструментальном индентировании. - Часть 1 : метод испытаний. Введ. 2013-05-01. - М. : Стандартинформ. - 2013. - С. 24.

11. Работнов Ю. Н. Ползучесть элементов конструкций. - М. : Наука, 1966. - 752 с.

Smirnov S.V. et al. / Studying epoxy resin reinforced with silica dioxide nanoparticles 
12. Малинин Н. Н. Прикладная теория пластичности и ползучести. - М. : Машиностроение, 1975. $-400 \mathrm{c}$.

13. Fischer-Cripps A. C. A simple phenomenological approach to nanoindentation creep // Materials Science and Engineering A. - 2004. - Vol. 385, iss. 1-2. - P. 74-82. DOI: 10.1016/j.msea.2004.04.070.

14. Natsik V. D., Fomenko L. S., Lubenets S. V. Investigation of the creep and glass transition of elastomers by the microindentation method: Epoxy resin and related nanocomposites // Physics of the Solid State. - 2013. - Vol. 55, no. 5. - P. 1020-1033. - DOI: 10.1134/S1063783413050260.

15. Биргер И. А., Мавлютов Р. Р. Сопротивление материалов : учебное пособие. М. : Наука. Гл. ред. физ.-мат. лит., 1986. - 560 с. 